RESEARCH ARTICLE

\title{
Estimation of Naringenin in Salacia reticulata Wight, Fishing Targets and Harmonizing the Ligand for Type 2 Diabetes
}

\author{
Anurabh Chakravarty ${ }^{1}$, Gnanam $\mathbf{R}^{2^{*}}$, Suresh $\mathrm{J}^{3}$ and Santhanakrishnan V $\mathbf{P}^{1}$ \\ ${ }^{1}$ Department of Plant Biotechnology, Tamil Nadu Agricultural University, Coimbatore-641 003 \\ ${ }^{* 2}$ Department of Plant Molecular Biology and Bioinformatics, Tamil Nadu Agricultural University, Coimbatore-641 003 \\ ${ }^{3}$ Department of Medicinal and Aromatic Plants, Tamil Nadu Agricultural University, Coimbatore-641 003
}

\begin{abstract}
Type 2 diabetes mellitus is characterized by insulin resistance and hyperglycemia and accounts for 90 per cent of diabetes cases. Salacia reticulata Wight, a climbing shrub indigenous to India and Sri Lanka, is an

Received : 03 ${ }^{\text {rd }}$ August, 2020 important source of anti-diabetic compounds. In this study, the abundance

Revised : $19^{\text {th }}$ August, 2020 of naringenin was estimated in various tissues of the plant using RP-HPLC.

Accepted : $11^{\text {th }}$ September, 2020 The highest concentration of naringenin was found in fresh and dried roots at 3.4 and $3.2 \%$, respectively. Through in silico analysis affinity of naringenin to PPAR- $\gamma, 11 \beta$-Hydroxysteroid-Dehydrogenase I, PPAR- $\alpha$, PPAR- $\delta$ and Glycogen Synthase Kinase - $3 \beta$, the targets for type 2 diabetes mellitus drug targets were $-8.7,-8.4,-7.1,-8.4$ and $-7.8 \mathrm{kcal} / \mathrm{mol}$, respectively.
\end{abstract}

Keywords: Naringenin; RP-HPLC; Type 2 diabetes mellitus; Salacia reticulata Wight.

\section{INTRODUCTION}

Diabetes mellitus is a major epidemic that is affecting lives adversely all around the world. This disease reduces the quality of life as well as lowers life expectancy (Tancredi et al., 2015). There are two major types of diabetes mellitus, type 1 and type 2 . Type 1 diabetes mellitus is an autoimmune disease and is a major cause of diabetes in childhood. In type 1 diabetes mellitus, the body's immune system attacks the insulin-producing beta cells in the pancreas. As a result, the body produces very little or no insulin. There is no known cure for type 1 diabetes mellitus. At the same time, type 2 diabetes mellitus accounts for the majority (around 90\%) of diabetes worldwide. Type 2 diabetes mellitus results in hyperglycemia as the body cannot respond to insulin, which is also termed as 'insulin resistance' (IDF Diabetes Atlas, 2019). People suffering from type 2 diabetes mellitus are at constant risk of developing life-threatening cardiovascular diseases, heart failure and chronic kidney disease. These diseases are associated with premature mortality, high rates of hospitalization and high healthcare costs. Type 2 diabetes mellitus is a major burden for healthcare systems the world over.

Traditionally plants have been used by several cultures all over the globe to treat diabetes. Salacia reticulata Wight is a large woody climbing shrub found in South Indian states ranging from Southern Odisha to Kerala as well as Sri Lanka. It is commonly called 'Ponkoranti' in Tamil, 'Ekanayakam' in Malayalam, 'Anukudu cettu' in Telegu, 'Saptaragni'

*Corresponding author's e-mail: gnanam.r@tnau.ac.in in Sanskrit and 'Kothala himbutu' in Sinhala. The roots and stem of Salacia reticulata Wight have been widely used in treating diabetes and obesity, gonorrhea and rheumatism, skin diseases and hemorrhoids (Im et al., 2008; Li et al., 2008; Matsuda et al., 2002; Nadkarni 1993)high-fat (HF).

Several anti-diabetic compounds have been identified in Salacia reticulata Wight, such as Mangiferin, kotalanol and salacinol (Yoshikawa et al., 1997; 2001; 1998). This plant is blessed with another major phytochemical, naringenin that also possesses anti-diabetic activity. The therapeutic potential of naringenin is well documented and its anti-diabetic properties are scrutinised (Salehi et al., 2019; Hartogh and Tsiani 2019). Naringenin potentiates intracellular signalling responses to low insulin doses by sensitizing hepatocytes to insulin. Naringenin although has been reported in another related species of Salacia, Salacia oblonga, studies on quantification of naringenin available in various tissues of Salacia reticulata Wight was not ventured. Naringenin content has been estimated in grape fruit by RP-HPLC, as a tool for quality control (Ribeiro and Ribeiro, 2008).

PPAR- - , PPAR- $\alpha$ and PPAR- $\delta$ are a group of ligandactivated transcription factors from the nuclear receptor super family (Peroxisome ProliferatorActivated Receptors (PPAR) that controls the expression of various genes entangled in glucose and lipid metabolism. The role of PPAR agonists is very important in managing metabolic disorders, but the existing PPAR agonists have undesired

$107|7-9| 287$ 
effects and researchers get going to focus on the discovery of new PPAR modulators that are safer, more beneficial without any undesired side effects. It has been hypothesized that increased $11 \beta$ HSD1 activity has a contributory role in the development of type 2 diabetes and $11 \beta$ HSD 1 inhibitors targeting metabolic syndrome, will be most useful in those with increased fasting blood glucose (Shukla et al., 2019). Glycogen synthase kinase-3 (GSK-3) has been targeted for the treatment of type 2 diabetes as GSK-3 plays in glucose homeostasis (MacAulay and Woodgett, 2008). The postulated functional role of GSK-3 in insulin signalling and glucose metabolism and that fact makes it a particularly intriguing candidate target for the treatment of type 2 diabetes (Nabben and Neumann, 2016). Keeping this in view, the targets have been short listed and an attempt was made to fill in that gap in knowledge, and also to investigate the activity of naringenin on various type 2 diabetes mellitus drug targets. The main motives for the study were to estimate the naringenin content in various tissues and to ascertain its anti-diabetic potential.

\section{MATERIAL AND METHODS}

\section{Reagents}

HPLC grade methanol, acetonitrile and naringenin standard (EC Number-266-769-1) were purchased from Sigma-Aldrich, St. Louis, MO, USA. Milli-Q ${ }^{\text {TM }}$ type 1 water was used for the study.

\section{RP-HPLC condition}

Shimadzu HPLC system equipped UV-Vis detector was used for RP-HPLC analysis. The compounds were separated using a $\mathrm{C}-18$ reversedphase column (INNO column, $5 \mu \mathrm{m}, 120 \AA$ A $4.6 \times$ $250 \mathrm{~mm}$ ). Shimadzu CLASS-VP ${ }^{\text {TM }}$ software was used for data acquisition, processing, and reporting on the Windows XP platform.

The isocratic mobile phase consisting of acetonitrile : water in 60 : 40 ratio was used. Detection of naringenin was done at $260 \mathrm{~nm}$ wavelength. A flow rate of $1 \mathrm{ml} \mathrm{min}{ }^{-1}$ was maintained. Naringenin was identified in samples based upon the retention time of the standard. The injection volumes was $10 \mu l$. The stimation was done using the formula:

1000 ppm solution was prepared by dissolving the naringenin standard in methanol. The stock solution was diluted to 100 ppm concentration for estimation of naringenin content in plant samples. The standard stocks were stored at $-20^{\circ} \mathrm{C}$.

\section{Plant samples}

Fresh plant samples (leaves and roots) of Salacia reticulata Wight were obtained from the Botanical Garden, Tamil Nadu Agricultural University, Coimbatore, India ( $\left.11^{\circ} 01^{\prime} 05.3^{\prime \prime} \mathrm{N} ; 76^{\circ} 55^{\prime} 58.1^{\prime \prime} \mathrm{E}\right)$.
Commercially available samples of root and root bark were obtained from sellers dealing in medicinal plant products.

\section{Preparation of plant samples}

$5 \mathrm{~g}$ of fresh were macerated using a pestle and mortar, the macerate was moved to Falcon tubes and $10 \mathrm{ml}$ of methanol was added to each. The tubes were placed on a shaker (C1 Platform Shaker, New Brunswick Scientific, Edison, NJ, USA). Extractions were carried out at room temperature with constant stirring at $110 \mathrm{rpm}$ for 24 hours. The methanolic extract was then filtered using a Whatman No. 1 filter paper and left to dry completely over 24 hours in cool and dry conditions. The solid remains were then weighed and reconstituted to make a 1000 ppm solution, which was diluted to $10 \mathrm{ppm}$ for analysis. The sample stocks were stored at $-20{ }^{\circ} \mathrm{C}$.

\section{Preparation of ligand library}

The structures of the identified compounds were downloaded from PubChem. The energy of the ligand was minimized using the UFF force field and folowing the steepest descent optimization algorithm.

\section{Preparation of target library}

The targets were chosen based on current drug targets for type 2 diabetes mellitus. The peroxisome proliferator-activated receptors (PPAR) are the targets for insulin-sensitizing thiazolidinedione drugs (Murphy and Holder, 2000). The enzyme $11 \beta$-Hydroxysteroid-Dehydrogenase type I plays a crucial role in modulating levels of glucocorticoids as it converts the inactive 11-keto glucocorticoids into active glucocorticoids. Excess glucorticoids has an adverse effect on glucose and lipid levels (Ge et al., 2010). Glycogen Synthase Kinase a serine/ threonine kinase plays a key role in insulin signaling and glucose metabolism. Glycogen Synthase Kinase inhibits the conversion of glucose to glycogen by binding to glycogen synthase. The inhibitors of Glycogen Synthase Kinase functions by mimicking insulin and thereby increase glycogen synthesis (Nabben and Neumann, 2016). The active residues and structure sources are mentioned in Table 1.

\section{Molecular Docking}

Naringenin was docked against the targets using MTiOpenScreen web-server (https://bioserv.rpbs. univ-paris-diderot.fr/services/MTiOpenScreen/) (Labbé et al., 2015). MTiOpenScreen performs binding site docking using AutoDock 4.2; the default exhaustiveness is set at 8 . The interacting residues were found using PLIP web-server (https://projects. biotec.tu-dresden.de/plip-web/plip/) (Salentin et al., 2015)drug discovery and biology. However, comprehensive tools are not freely available to the research community. Here, we present the proteinligand interaction profiler (PLIP). 


\section{RESULTS AND DISCUSSION}

\section{Tissue wise naringenin quantification}

The naringenin content was found to be highest in dried roots at $3.4 \%$, while it was slightly lower in fresh roots at $3.2 \%$. Significant concentrations of naringenin are also found in leaves at $0.8 \%$. Whereas in the root bark it is $0.28 \%$. The percentage content of naringenin in the various tissues have been visualized in Figure 1. The chromatograms for the samples have been displayed in Figure 2. The compound naringenin has been previously isolated from the related species Salacia oblonga and was shown to have potent anti-diabetic activity.

Table 1. The active residues for the various drug targets for type 2 diabetes mellitus

\begin{tabular}{|c|c|c|c|c|}
\hline S.No & PDB IDs & Target (Mode of action) & Active Residues & Sources \\
\hline 1 & $20 \mathrm{M9}$ & PPAR- (Agonist) & $\begin{array}{l}\text { LYS256, HIS266, SER342, PHE264, ILE281, } \\
\text { PHE282, GLY284, CYS285, VAL339, ILE341, } \\
\text { MET348, LEU353, LEU356 }\end{array}$ & (Ambrosio et al., 2007) \\
\hline 2 & 2ILT & $\begin{array}{l}11 \text {-Hydroxysteroid- } \\
\text { Dehydrogenase type I(Inhibitor) }\end{array}$ & THR124, LEU126, SER170, TYR177, TYR183 & $\begin{array}{l}\text { (Valeur et al., 2012; } \\
\text { Sorensen et al., 2007) }\end{array}$ \\
\hline 3 & 3ET1 & PPAR- (Agonist) & TYR314, TYR464, PHE318, HIS440, GLN277 & (Artis et al., 2009) \\
\hline 4 & $30 Z 0$ & PPAR- (Agonist) & THR289, HIS323, HIS449, TYR473 & (Luckhurst et al., 2011) \\
\hline 5 & $4 J 1 R$ & $\begin{array}{l}\text { Glycogen Synthase Kinase - } 3 \\
\text { (Inhibitor) }\end{array}$ & $\begin{array}{l}\text { ASP133, ILE62, GLY63, PHE67, VAL70, } \\
\text { VAL110, LEU132, TYR134, VAL135, PRO136, } \\
\text { LEU188, CYS199, ASP200 }\end{array}$ & \\
\hline
\end{tabular}

The compound was able to reduce blood glucose levels in streptozotocin-induced diabetic rats (Singh et al., 2017).

\section{Molecular docking analysis}

The anti-diabetic potential of naringenin is an established fact, but the mechanism of its action is not. It has been suggested that naringenin binds to PPAR-y, but it could also potentially be having other modes of action (Singh et al., 2017). Naringenin showed the highest affinity for PPAR-y with a binding affinity of $-8.7 \mathrm{kcal} / \mathrm{mol}$. It forms three hydrogen bonds facilitated by the residues LYS265, HIS266 and ARG280.

Table 2. The binding affinity of naringenin and interacting residues for the different targets.

\begin{tabular}{|c|c|c|c|c|c|}
\hline S.No & Target & $\begin{array}{l}\text { Binding Affinity } \\
\text { (kcal/mol) }\end{array}$ & Hydrogen Bonds & $\begin{array}{l}\text { Hydrophobic } \\
\text { Interactions }\end{array}$ & Stacking \\
\hline 1 & PPAR- & -8.7 & LYS265, HIS266, ARG280 & HIS266 & \\
\hline 2 & $\begin{array}{l}11 \text {-Hydroxysteroid- } \\
\text { Dehydrogenase I }\end{array}$ & -8.4 & $\begin{array}{l}\text { LYS44, ILE46, GLY47, ILE121, } \\
\text { ILE218 }\end{array}$ & ILE46, ILE218 & \\
\hline 3 & PPAR- & -7.1 & $\begin{array}{l}\text { GLN277, SER280, HIS440, } \\
\text { HIS } 457\end{array}$ & $\begin{array}{l}\text { PHE273, VAL444, } \\
\text { LEU460 }\end{array}$ & \\
\hline 4 & PPAR- & -8.4 & PHE327, HIS449 & $\begin{array}{l}\text { PHE327, LEU330, } \\
\text { VAL334, LEU339, } \\
\text { ILE364, LYS367 }\end{array}$ & HIS449 \\
\hline 5 & $\begin{array}{l}\text { Glycogen Synthase } \\
\text { Kinase - } 3\end{array}$ & -7.8 & $\begin{array}{l}\text { ILE62, PHE67, VAL70, ALA83, } \\
\text { LEU132 }\end{array}$ & $\begin{array}{l}\text { ASN64, ASP133, } \\
\text { VAL135, ASP200, } \\
\text { GLY262 }\end{array}$ & \\
\hline
\end{tabular}

The binding affinity for $11 \beta$-HydroxysteroidDehydrogenase type I is $-8.4 \mathrm{kcal} / \mathrm{mol}$, while forming five hydrogen bonds with the residues are LYS44, ILE46, GLY47, ILE121, ILE218. The binding affinity for naringenin with PPAR- $\alpha$ and PPAR- $\delta$ are -7.1 and $-8.4 \mathrm{kcal} / \mathrm{mol}$. In contrast, the binding affinity for Glycogen Synthase Kinase is - $7.8 \mathrm{kcal} /$ mol. The binding affinities of naringenin for the various receptors have been elucidated in Table 2 . The binding pockets have been visualized in Figure 3. The residues ILE62 and VAL135 seem to be crucial for interacting with inhibitors. In a study conducted to find the inhibitory effects of the compound olanzapine, the compound formed hydrogen bonds with Glycogen Synthase Kinase via ILE62 and VAL135, while also having hydrophobic interactions with residues ALA83, VAL110 and LEU132 (Mohammad et al., 2007).

For this study, RP-HPLC technique was used to quantify the amount of naringenin in various tissues. In Salacia oblonga NMR based metabolomics analysis was used to identify naringenin (Singh et al., 2017). While in silico study was done to identify the anti-diabetic potential of naringenin. From the 


\section{Naringenin content}

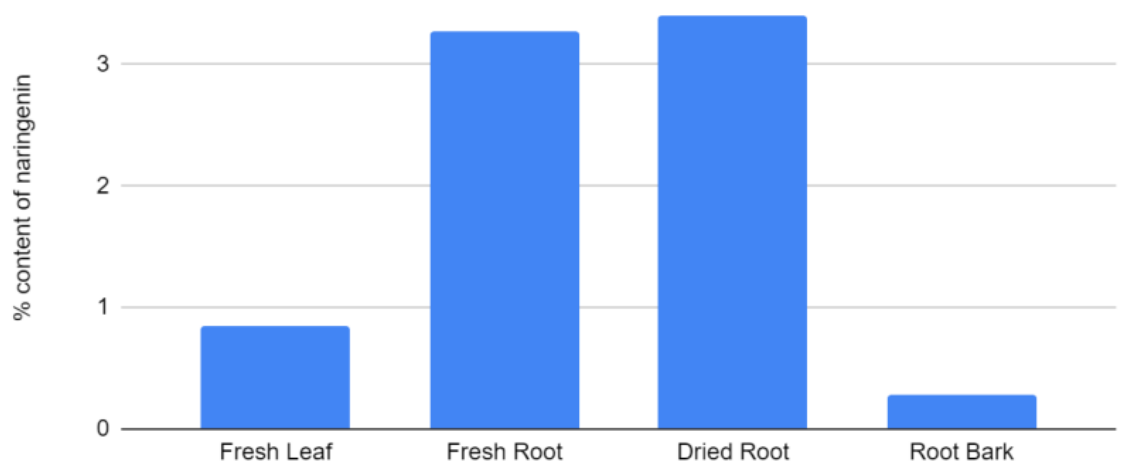

Figure 1. Naringenin content in various tissues of Salacia reticulata Wight.

study, it was found that the highest concentration of naringenin was found in the roots (fresh and dried) of Salacia reticulata Wight. Though the presence of naringenin in observed in the leaves and the root bark, the quantity is supplementary. The affinity of naringenin is highest for PPAR-y which is consistent with past findings (Singh et al., 2017; Rigano et al., 2017). The PPARs are isotypes encoded by separate
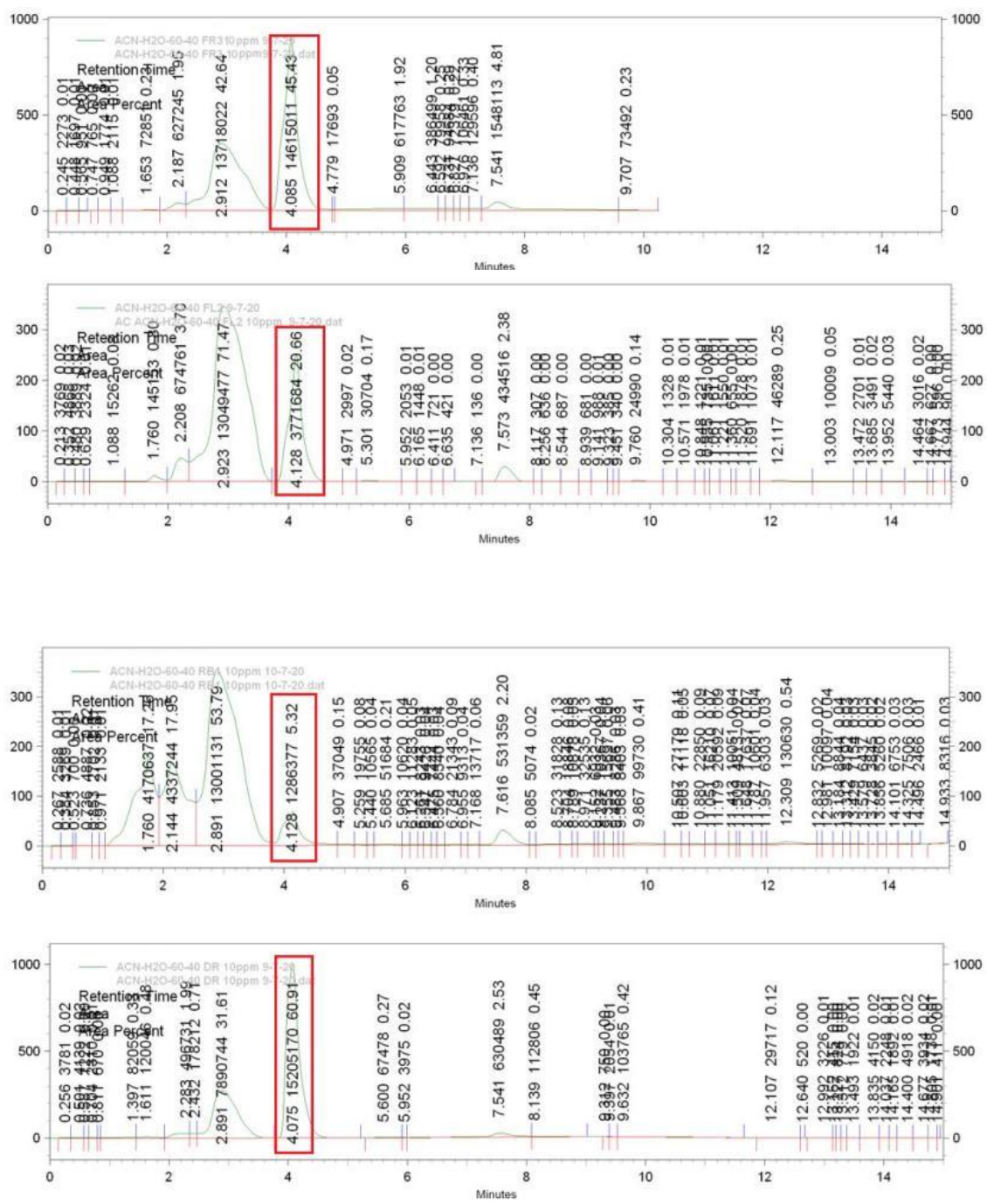

Figure 2. Naringenin estimation chromatograms for: 1 Fresh Leaf; 2 Fresh Root; 3 Dried Root; 4 Root Bark. 
genes, which share a high degree of sequence similarity and structural homology. The ability of naringenin to bind to PPAR- $\alpha$ and PPAR- $\delta$ has been suggested due to their structural similarity to PPAR-y (Rigano et al., 2017). Naringenin as an inhibitor of
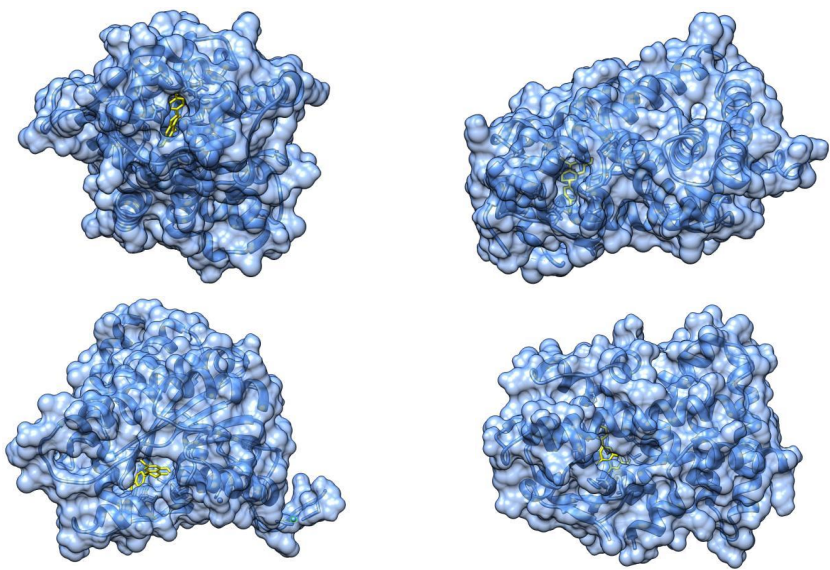

$11 \beta$-Hydroxysteroid-Dehydrogenase type I has also been studied before through in vitro studies on the enzyme obtained from guinea pig kidneys (Zhang et al., 1994).

Figure 3. Docked poses of naringenin with the following receptors: 1PPAR-V; 2 11ß-HydroxysteroidDehydrogenase type I; 3 PPAR- $\alpha$; 4 PPAR- $\delta$; 5Glucosekinase Regulatory Protein.

Though the effects of naringenin is well studied, it includes lowering of lipid peroxidation biomarkers, protein carbonylation induces carbohydrate metabolism scavenges for reactive oxygen species, modulates immune system activity, and also exerts anti-atherogenic and anti-inflammatory effects (Salehi et al., 2019; Wang et al., 2015). Studies such as X-ray crystallography are required to further validate the binding modes of naringenin to the targets.

\section{Funding and Acknowledgment}

The authors acknowledge the financial support in the form of fellowship provided to AC by DBT, Gol.No. BT/HRD/01/011/88- Vol-VI dt.06.11.2019 (San. No. 102/IFD/SAN/2612/2019-20 dt. 05.11.2019)

\section{Ethics statement}

No specific permits were required for the described field studies because no human or animal subjects were involved in this research.

\section{Consent for publication}

All the authors agreed to publish the content.

\section{Competing interests}

There were no conflict of interests in the publication of this content

\section{Author contributions}

Research grant - AC; Idea conceptualization - RG; Experiments - AC; Guidance - RG, SVP, JS; Writingoriginal draft - AC; Writing- reviewing \& editing- RG

\section{REFERENCES}

Atlas, IDF Diabetes. "9th updated the 2019 edition.

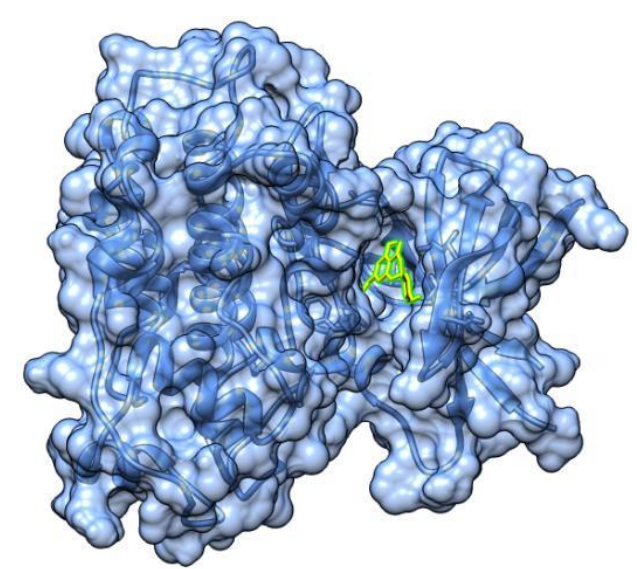

International Diabetes Federation." (2020).

Ambrosio, Andre L.B., Sandra M.G. Dias, Igor Polikarpov, Robert B. Zurier, Sumner H. Burstein, and Richard C. Garratt. 2007. "Ajulemic Acid, a Synthetic Nonpsychoactive Cannabinoid Acid, Bound to the Ligand Binding Domain of the Human Peroxisome Proliferator-Activated Receptor ." Journal of Biological Chemistry 282 (25): 18625-33.

Artis, Dean R., Jack J. Lin, Chao Zhang, Weiru Wang, Upasana Mehra, Mylene Perreault, David Erbe, et al., 2009. "Scaffold-Based Discovery of Indeglitazar, a PPAR Pan-Active Anti-Diabetic Agent." Proceedings of the National Academy of Sciences of the United States of America 106 (1): 262-67.

Ge R, Huang Y, Liang G, Li X. 11beta-hydroxysteroid dehydrogenase type 1 inhibitors as promising therapeutic drugs for diabetes: status and development. Curr. Med. Chem., 2010 17(5):41222.

Hartogh, Danja J.Den and Evangelia Tsiani. 2019. "Antidiabetic Properties of Naringenin: A Citrus Fruit Polyphenol." Biomolecules 9(3): 4-9.

Im, Ryanghyok, Hiroshi Mano, Sachie Nakatani, Jun Shimizu, and Masahiro Wada. 2008. "Aqueous Extract of Kotahla Himbutu (Salacia Reticulata) Stems Promotes Oxygen Comsumption and Supresses Body Fat Accumulation in Mice." JOURNAL OF HEALTH SCIENCE 54(6): 645-53.

Labbé, Céline M., Julien Rey, David Lagorce, Marek Vavru a, JéRome Becot, Olivier Sperandio, Bruno O. Villoutreix, Pierre Tufféry, and Maria A. Miteva. 2015. "MTiOpenScreen: A Web Server for Structure-Based Virtual Screening." Nucleic Acids Research 43 (W1): W448-54.

Li, Yuhao, Tom Hsun-Wei Huang, and Johji Yamahara. 2008. "Salacia Root, a Unique Ayurvedic Medicine, Meets Multiple Targets in Diabetes and Obesity." Life Sciences 82 (21-22): 1045-49. 
Luckhurst, Christopher A., Linda A. Stein, Mark Furber, Nicola Webb, Marianne J. Ratcliffe, Gary Allenby, Sara Botterell, Wendy Tomlinson, Barrie Martin, and Andrew Walding. 2011. "Discovery of Isoindoline and Tetrahydroisoquinoline Derivatives as Potent, Selective PPAR Agonists." Bioorg. Med. Chem. Lett ., 21 (1): 492-96.

MacAulay, Katrina, and James R. Woodgett. 2008. "Targeting Glycogen Synthase Kinase-3 (GSK-3) in the Treatment of Type 2 Diabetes." Expert Opin Ther Targets., 12 (10): 1265-74.

Matsuda, Hisashi, Toshio Morikawa, and Masayuki Yoshikawa. 2002. "Antidiabetogenic Constituents from Several Natural Medicines." Pure Appl. Chem., 74 (7): 1301-8.

Mohammad, Mohammad K., Ihab M. Al-masri, Mutasem O. Taha, Mohamed A.S. Al-Ghussein, Hatim S. AlKhatib, Samer Najjar, and Yasser Bustanji. "Olanzapine Inhibits Glycogen Synthase Kinase3: An Investigation by Docking Simulation and Experimental Validation." Eur. J. Pharmacol., 584 (1): 185-91.

Murphy, Gregory J, and Julie C Holder. "PPAR- Agonists: Therapeutic Role in Diabetes, Inflammation and Cancer." Trends in Pharmacological Sciences 21(12): 469-74.

Nabben, Miranda, and Dietbert Neumann. 2016. "GSK3 Inhibitors: Anti-Diabetic Treatment Associated with Cardiac Risk?: Editorial to: 'The Impact of Chronic Glycogen Synthase Kinase-3 Inhibition on Remodeling of Normal and Pre-Diabetic Rat Hearts.' by Barbara Huisamen et al.," Cardiovascular Drugs and Therapy 30 (3): 233-35.

Nadkarni, K M. 1993. The Indian Materia Medica. Vol. 1: 1089. Bombay: Popular Prakashan Pvt. Ltd.

Ribeiro, Isabel A., and Maria H.L. Ribeiro. "Naringin and Naringenin Determination and Control in Grapefruit Juice by a Validated HPLC Method." Food Control 19(4): 432-38.

Rigano, Daniela, Carmina Sirignano, and Orazio Taglialatela-Scafati. 2017. "The Potential of Natural Products for Targeting PPAR ." Acta Pharmaceutica Sinica $B 7$ (4): 427-38.

Salehi, Bahare, Patrick Valere Tsouh Fokou, Mehdi Sharifi-Rad, Paolo Zucca, Raffaele Pezzani, Natália Martins, and Javad Sharifi-Rad. 2019. "The Therapeutic Potential of Naringenin: A Review of Clinical Trials." Pharmaceuticals 12 (1): 1-18.

Salentin, Sebastian, Sven Schreiber, V. Joachim Haupt, Melissa F. Adasme, and Michael Schroeder. 2015. "PLIP: Fully Automated Protein-Ligand Interaction Profiler." Nucleic Acids Research 43 (W1): W443-47.

Shukla, Ravindra, Asish Kumar Basu, Biplab Mandal, Pradip Mukhopadhyay, Animesh Maity, Satyam Chakraborty, and Praveen Kumar Devrabhai. 2019.
"11 Hydroxysteroid Dehydrogenase - 1 Activity in Type 2 Diabetes Mellitus: A Comparative Study." BMC Endocrine Disorders 19 (1): 1-9.

Singh, Ashok K., Vinit Raj, Amit K. Keshari, Amit Rai, Pranesh Kumar, Atul Rawat, Biswanath Maity, et al., 2017. "Isolated Mangiferin and Naringenin Exert Antidiabetic Effect via PPAR /GLUT4 Dual Agonistic Action with Strong Metabolic Regulation." ChemicoBiological Interactions 280: 33-44.

Sorensen, Bryan, Martin Winn, Jeff Rohde, Qi Shuai, Jiahong Wang, Steven Fung, Katina Monzon, et al., 2007. "Adamantane Sulfone and Sulfonamide 11- -HSD1 Inhibitors." Bioorg. Med. Chem. Lett ., 17 (2): 527-32.

Tancredi, Mauro, Annika Rosengren, Ann Marie Svensson, Mikhail Kosiborod, Aldina Pivodic, Soffia Gudbjörnsdottir, Hans Wedel, Mark Clements, Sofia Dahlqvist, and Marcus Lind. 2015. "Excess Mortality among Persons with Type 2 Diabetes." N. Engl. J. Med., 373 (18): 1720-32.

Valeur, Eric, Serge Christmann-Franck, Franck Lepifre, Denis Carniato, Daniel Cravo, Christine Charon, Sophie Bozec, et al., 2012. "Structure-Based Design of 7-Azaindole-Pyrrolidine Amides as Inhibitors of 11 -Hydroxysteroid Dehydrogenase Type I." Bioorg. Med. Chem. Lett 22 (18): 5909-14.

Yoshikawa, Masayuki, Toshiyuki Murakami, Hiromi Shimada, Hisashi Matsuda, Johji Yamahara, Genzou Tanabe, and Osamu Muraoka. 1997. “Salacinol, Potent Antidiabetic Principle with Unique Thiosugar Sulfonium Sulfate Structure from the Ayurvedic Traditional Medicine Salacia Reticulata in Sri Lanka and India." Tetrahedron Letters 38 (48): 8367-70.

Yoshikawa, Masayuki, Toshiyuki Murakami, Kenichi Yashiro, and Hisashi MATSUDA. 1998. "Kotalanol, a Potent -Glucosidase Inhibitor with Thiosugar Sulfonium Sulfate Structure, from Antidiabetic Ayurvedic Medicine Salacia Reticulata." Chemical and Pharmaceutical Bulletin 46 (8): 1339-40.

Yoshikawa, Masayuki, N Nishida, H Shimoda, M Takada, Y Kawahara, and H Matsuda. 2001. "Polyphenol Constituents from Salacia Species: Quantitative Analysis of Mangiferin with Alpha-Glucosidase and Aldose Reductase Inhibitory Activities." Yakugaku Zasshi: Journal of the Pharmaceutical Society of Japan 121 (5): 371.

Zhang, Yin Di, Beverly Lorenzo, and Marcus M. Reidenberg. 1994. "Inhibition of 11 -Hydroxysteroid Dehydrogenase Obtained from Guinea Pig Kidney by Furoemide, Naringenin and Some Other Compounds." J.Steroid Biochem. Mol. Biol 49 (1): 81-85. 